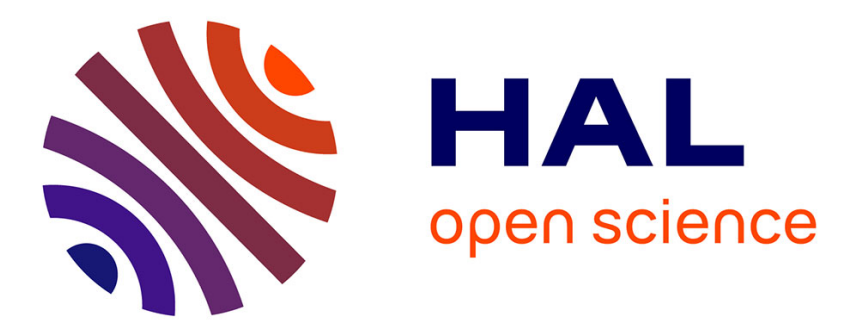

\title{
An electron holography study of perpendicular magnetic tunnel junctions nanostructured by deposition on pre-patterned conducting pillars
}

V. Boureau, V. D Nguyen, Aurélien Masseboeuf, A. Palomino, E Gautier, J. Chatterjee, S. Lequeux, S. Auffret, L. Vila, R. Sousa, et al.

\section{To cite this version:}

V. Boureau, V. D Nguyen, Aurélien Masseboeuf, A. Palomino, E Gautier, et al.. An electron holography study of perpendicular magnetic tunnel junctions nanostructured by deposition on pre-patterned conducting pillars. Nanoscale, 2020, 12 (33), pp.17312-17318. 10.1039/D0NR03353G . hal-02953621

\section{HAL Id: hal-02953621 \\ https://hal.science/hal-02953621}

Submitted on 30 Sep 2020

HAL is a multi-disciplinary open access archive for the deposit and dissemination of scientific research documents, whether they are published or not. The documents may come from teaching and research institutions in France or abroad, or from public or private research centers.
L'archive ouverte pluridisciplinaire HAL, est destinée au dépôt et à la diffusion de documents scientifiques de niveau recherche, publiés ou non, émanant des établissements d'enseignement et de recherche français ou étrangers, des laboratoires publics ou privés. 
Received 00th January 20xx, Accepted 00th January 20xx DOI: $10.1039 / \times 0 \times x 00000 x$

\title{
An electron holography study of perpendicular magnetic tunnel junctions nanostructured by deposition on pre-patterned conducting pillars
}

\begin{abstract}
V. Boureau, ${ }^{a}$ V.D. Nguyen, ${ }^{b}$ A.Masseboeuf, ${ }^{b}$ A. Palomino, ${ }^{b}$ E. Gautier, ${ }^{b}$ J. Chatterjee, ${ }^{b}$ S.Lequeux, ${ }^{b}$ S. Auffret, ${ }^{b}$ L. Vila, ${ }^{b}$ R. Sousa, ${ }^{b}$ L. Prejbeanu, ${ }^{b}$ D. Cooper ${ }^{a}$ and B. Dieny ${ }^{b}$

Abstract: The fabrication of muti-gigabit magnetic random access memory (MRAM) chips requires the patterning of magnetic tunnel junctions at very small dimensions (sub-30 nm) and very dense pitch. This remains a challenge due to the difficulty in etching magnetic tunnel junction stacks. We earlier proposed a strategy to circumvent this problem by depositing the magnetic tunnel junction material on prepatterned metallic pillars, the junction being then naturally shaped during deposition. Once electrically contacted, the deposit on top of the pillars constitutes the magnetic storage element of the memory cell. However, in this process, magnetic material is also deposited in the trenches between the pillars that might affect the memory cell behaviour. Here we study the magnetic interactions between the deposit on top of the pillars and in the trenches by electron holography, at room temperature and up to $325^{\circ} \mathrm{C}$. Supplied by models, we show that the additional material in the trenches is not perturbing the working principle of the memory and even plays a role of flux absorber which reduces the crosstalk between neighboring dots. Besides, in the studied sample, the magnetization of the $1.4 \mathrm{~nm}$ thick storage layer of the dots is found to switch from out-of-plane to an in-plane configuration above $125{ }^{\circ} \mathrm{C}$, while gradually decreasing with temperature. Electron holography is shown to constitute a very efficient tool for characterizing the micromagnetic configuration of the storage layer in MRAM cells.
\end{abstract}

Kewords: Magnetic tunnel junction, MRAM, Electron holography, Temperature, Magnetic field

\section{Introduction}

Spin transfer torque magnetic random access memory (STTMRAM) based on out-of-plane magnetized perpendicular magnetic tunnel junction (pMTJ) is one of the most promising technologies of emerging non-volatile memories [1]. Indeed, it possesses a unique combination of assets: quasi-infinite write endurance ( $>10^{12}$ cycles at $30 \mathrm{~ns}$ pulse length), high speed (write pulse down to $1 \mathrm{~ns}$ ) [2], low power consumption (in the range of tens of $\mathrm{fJ}$ ) [1], high density (4 Gbit chip demonstrated [3]) and scalability [4, 5]. Embedded STT-MRAM are entering in volume production for embedded Flash (eFlash) replacement and last level cache applications. For this type of applications not requiring very high memory density, the preferred etching technique is still ion beam etching (IBE) [1]. However, this technique is not appropriate for very high density memory due to shadowing effects. This effect worsens as the memory pitch shrinks typically below 5 times the feature size (5F) resulting in a poor control of the critical dimension at very narrow pitch [6]. In addition, it is difficult to implement IBE on large wafers with good uniformity [6]. Reactive ion etching (RIE) was also

\footnotetext{
a. Univ. Grenoble Alpes, CEA-LETI, F-38000 Grenoble, France

${ }^{b}$ SPINTEC, Univ. Grenoble Alpes, CEA, CNRS, F-38000 Grenoble, France
}

investigated for MTJs patterning with various gases but was found to be very complex due to the heterogeneous nature of the MTJ stacks and to cause corrosion and delamination of the magnetic materials [7]. Therefore an alternative method for nano-patterning MTJ elements at small feature size $(F<20 \mathrm{~nm})$ and narrow pitch $(p \approx 2 F)$ is still required to enable the fabrication of dense STT-MRAM arrays. For this purpose, we recently proposed a novel approach for the patterning of the MTJ stacks [8]. It consists in depositing the MTJ material by physical vapor deposition (PVD) on arrays of pre-patterned conducting pillars. Those are made of a non-magnetic material for which the patterning process is already well controlled (e.g. Ta pillars prepared by RIE, or $\mathrm{Cu}$ or $\mathrm{W}$ vias prepared by damascene process) [8]. In this way, the MTJ stack is naturally structured while being deposited and no post-deposition etching is required. Note that during deposition, part of the magnetic material is also deposited in the trenches between pillars. However, this residual magnetic material does not hinder the working principle of the STT-MRAM provided it does not induce electrical short circuits between neighboring pillars. These short circuits are avoided by giving to the prepatterned pillars a T-shape in cross-section with undercuts so that no residual material is deposited at the foot of the pillars. This geometry can be seen in Figure 1. 
In this study, we investigate the magnetic properties of arrays of memory cells prepared by this method. We show that electron holography is a very efficient characterization technique to image the stray fields generated by these magnetic memory devices and from this, derive information on their micromagnetic configuration and interactions. In particular, the magnetostatic interactions between neighboring cells and notably between the magnetic deposit on top of the pillars and in the trenches between pillars was investigated. To better reveal the influence of these magnetostatic interactions, we used MTJ stacks with relatively weak perpendicular anisotropy and coercivity compared to those of conventional STT-MRAM (coercive field in the present devices around $15 \mathrm{mT}$ instead of $200 \mathrm{mT}$ in conventional STTMRAM). An antiparallel coupling of the magnetization on top of the pillars and in the trenches at the foot of the pillars was shown. Hence the deposit in the trenches is found to play the role of a flux absorber reducing the magnetostatic interactions and therefore the crosstalk between neighboring cells. In addition, we investigated the behavior of the magnetic configuration in-situ with temperature using holography and showed that a reorientation of anisotropy from out-of-plane to in-plane takes place around $125^{\circ} \mathrm{C}$ accompanied by a gradual decrease of the magnetization.

\section{Specimens and methods}

\section{Specimens}

The experiments were conducted on arrays of magnetic dots obtained by depositing MTJ stacks on pre-patterned T-shape $\mathrm{Ta} / \mathrm{Pt}$ pillars [8]. As previously mentioned, the purpose of this T-shape is to avoid electrical short circuits between neighboring pillars which would be caused by electrical contact between the foot of the non-magnetic metallic pillars and the metallic deposit in the trenches. Besides, the pillar height must be larger than the total thickness of the deposited stack to insure electrical insulation between the deposit in the trenches and on top of the pillar. Figures 1(a) and 1(b) show an array of metallic T-shaped pillars before and after deposition of the magnetic stack respectively. After deposition of the magnetic stack, one can see that there is indeed no metallic deposit surrounding the foot of the non-magnetic metallic pillar.

The details of the fabrication process are given in [8], only the main steps are described here. The non-magnetic metallic pillars consist of a Ta foot and a Pt cap. The Pt cap and Ta foot are first etched by anisotropic RIE, next the Ta foot is trimmed by isotropic RIE to produce the T-shape. This process allowed us to fabricate arrays of pillars of diameter down to $F=35 \mathrm{~nm}$ with a pitch down to $p=1.5 F$. The MTJ magnetic stack was then deposited by sputtering on this pre-patterned substrate and consists of the following layers: a compensated synthetic antiferromagnetic (SyAF) layer acting as a hard reference, $\mathrm{MgO}$ barrier, $1.4 \mathrm{~nm}$ of FeCoB (storage layer), $1 \mathrm{~nm}$ of Ta and $2 \mathrm{~nm}$ of Pt. All the magnetic layers exhibit out-of-plane magnetic anisotropy. The MTJ is naturally patterned during its deposition on the pre-existing pillars. Figure 1 (b) clearly shows the magnetic deposit on top of the metallic pillars and in the trenches between them. Some rounding of the deposit can be seen at the edges of the pillar in Figure 1 (d) which results from the fact that the material was deposited by sputtering which is not a very directional technique. Nevertheless, our earlier study indicated that the continuity of the $\mathrm{MgO}$ barrier is preserved to the very edge of the pillar thus avoiding short circuits between top and bottom magnetic electrodes across the tunnel barrier [8]. Spin-transfer torque switching has been demonstrated in STT-MRAM cells prepared by this technique [8]. The present study focuses on the magnetic properties of these arrays of dots. Therefore the fabrication process was stopped just after the deposition of the MTJ stack on the prepatterned pillars.
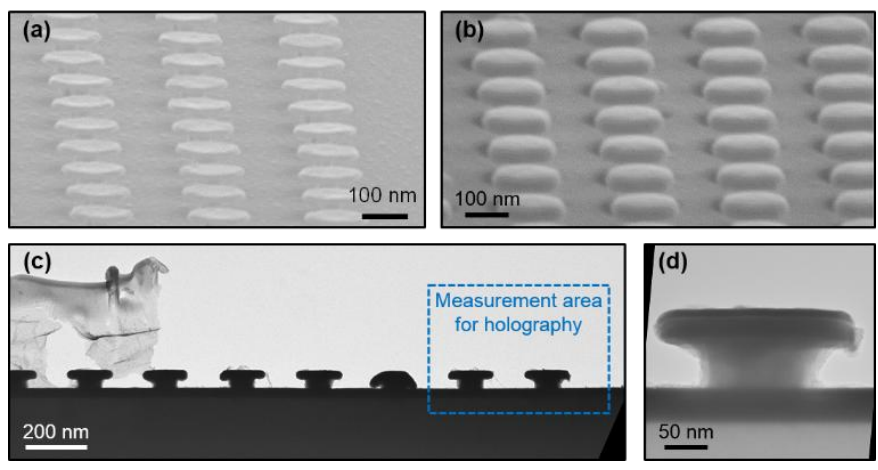

Figure 1: Scanning electron microscopy images of an array of T-shaped Ta/Pt metallic pillars of top diameter $150 \mathrm{~nm}$ and pitch $260 \mathrm{~nm}$ (a) before and (b) after deposition of the MTJ stack. (c) Cross-section transmission electron microscopy bright field image of a sample of pillars of top diameter $230 \mathrm{~nm}$ and pitch $400 \mathrm{~nm}$ coated with the magnetic stack, with indication of the region where holography observations were performed. (d) Enlargement of the pillar located on the right-side of image (c).

\section{Holography}

For the transmission electron microscopy (TEM) experiments, it was important to observe only one row of pillars in projection that had not been exposed to $\mathrm{Ga}^{+}$ions during focused ion beam (FIB) milling. A specimen was prepared from the bulk sample with pillars diameters of $230 \mathrm{~nm}$, pitch $400 \mathrm{~nm}$ and height $90 \mathrm{~nm}$. The surface of the bulk sample was protected from the $\mathrm{Ga}^{+}$ion implantation by a deposit of an organic ink a few hundreds of nanometers thick. A Zeiss NVision 40 dual-beam equipment operated at $30 \mathrm{kV}$ was then used to extract the region of interest which was then attached to a TEM grid support and thinned down to about $300 \mathrm{~nm}$. Finally, the protective ink was partially removed by an $\mathrm{Ar} / \mathrm{O}_{2}$ plasma clean with the rest mechanically removed using an Omniprobe system.

Off-axis electron holography [9] experiments were performed on a double aberration-corrected FEI Titan Ultimate TEM operated at $200 \mathrm{kV}$ acceleration voltage in Lorentz mode. Here an electron biprism is used to interfere an electron wave that passes through the region of interest with a wave that passes through a field free region to form an interference pattern known as a hologram. A Fourier reconstruction routine is then used to reconstruct a phase image from the hologram, with a spatial resolution of $8.5 \mathrm{~nm}$. Spatial resolutions of better than 
$1 \mathrm{~nm}$ are possible using this technique, but here the experiment was optimised to have a large field of view. In this study, series of holograms were used to provide very long cumulative exposure times, of $512 \mathrm{~s}$, which increases the signal-to-noise ratio of the quantitative phase images [10].

The phase images are sensitive to both magnetic and electric contributions of the sample. To remove the electrostatic contributions arising from residues of ink charging under the electron beam, a well-known method is used where the holography is performed twice, before and after turning over the specimen inside the sample holder of the TEM [11]. As the electrostatic contribution to the phase originates from a scalar field and the magnetic contribution from a vector field, the difference of the two phase images is twice the magnetic phase contribution, $\varphi^{M}$, expressed as follows:

$$
\varphi^{M}=-\frac{e}{\hbar} \int A_{z} d z
$$

$e$ is the electron charge, $\hbar$ the reduced Planck constant, $z$ the direction of propagation the electron beam and $A_{z}$ the zcomponent of the magnetic vector potential. Hence the magnetic phase map measured by holography is sensitive to the magnetic vector potential component that is parallel to the electron beam direction and integrated over the electron beam path.

Analysis of the magnetic phase maps provides detailed information on the magnetic configuration of the sample. The cosine of the magnetic phase image depicts the isophase lines, corresponding to the magnetic flux lines, that are projected over the electron beam path. Vector magnetic induction maps can be calculated from the gradient of the magnetic phase, considering that

$$
\vec{B}=\vec{\nabla} \times \vec{A}
$$

where $\vec{B}$ is the magnetic induction and $\vec{\nabla} \times$ the rotational operator. Using an appropriate (Coulomb) gauge, the quantitative projection of the magnetic induction over the electron path is given by:

$$
\int \overrightarrow{B_{\perp}} d z=-\frac{\hbar}{e} \vec{\nabla} \times \varphi^{M}
$$

where $\overrightarrow{B_{\perp}}$ corresponds to the components of the magnetic induction that are perpendicular to the propagation direction of the electron beam.

To complement the experiments, phase images were simulated, starting from a guessed magnetization map of the system in 2D. The two components of magnetization perpendicular to the electron beam are summed over $z$ and the last component of magnetization parallel to the electron beam is neglected.

For temperature in-situ holography measurements, a Gatan heating holder was used. It then becomes problematic to turn over the specimen at each temperature, in order to separate the magnetic from the electric phase. Therefore, the magnetic phase from a first experiment, acquired at room temperature and labelled $\varphi_{R T r e f}^{M}$, was used as a reference to remove the electrostatic potentials. The in-situ measurements contain both the electric and magnetic phase contributions, $\varphi_{\theta}=\varphi_{\theta}^{E}+\varphi_{\theta}^{M}$, where $\theta$ is the temperature of the measurement. As the sample remains unmodified at the start of the in-situ experiment, the magnetic phase contribution at room temperature is the same as the reference, $\varphi_{R T}^{M}=$ $\varphi_{\text {RTref }}^{M}$. Thus considering that the electric phase contribution is constant during the in-situ experiment, $\varphi_{R T}^{E}=\varphi_{\theta}^{E}$, it becomes possible to extract the magnetic phase contribution throughout the temperature in-situ experiment by combining three phase images:

$$
\varphi_{\theta}^{M}=\varphi_{\theta}-\varphi_{R T}+\varphi_{R T r e f}^{M}
$$

\section{Results and discussion}

\section{Magneto-optic Kerr effect}

A focused magneto-optic Kerr effect (MOKE) characterization was performed on a sample with pillar diameters $200 \mathrm{~nm}$, pitch $400 \mathrm{~nm}$ and height $80 \mathrm{~nm}$. Figure 2(a) shows a schematic where the laser beam spot of about $700 \mathrm{~nm}$ in diameter irradiated a few magnetic dots as well as the deposit between them. Figure 2(b) shows the measured hysteresis loops with field applied out-of-plane. Two magnetization jumps are clearly distinguished in the range of field used. They correspond to the switching fields of the free layer of the stack (storage layer) on top of the pillars and in the trenches. As usually observed, the coercive field of the patterned structures on top of the pillars is larger than that of the continuous film of same composition in the trenches. This difference in coercivity allows to unambiguously determine the switching field of the MTJ dots. This experiment also confirmed that the MTJs deposited on the pre-patterned pillars exhibit out-of-plane anisotropy as expected. The magnetic materials used in this model system are relatively soft since the coercivity of the deposit in the trenches is only $5.8 \mathrm{mT}$ whereas that on top of the dot is $11.6 \mathrm{mT}$. This relatively low coercivity comes from the chosen FeCoB thickness which is close to the critical thickness at which a reorientation of the anisotropy from outof-plane to in-plane anisotropy takes place [1]. The lower coercivity in the trenches than on top of the dots is due to the easy propagation of domain walls throughout the continuous film covering the trenches. When moving the laser spot across the sample, the dot to dot variability in the coercive field of the deposit on top of the dot is estimated to be $\pm 20 \%$.

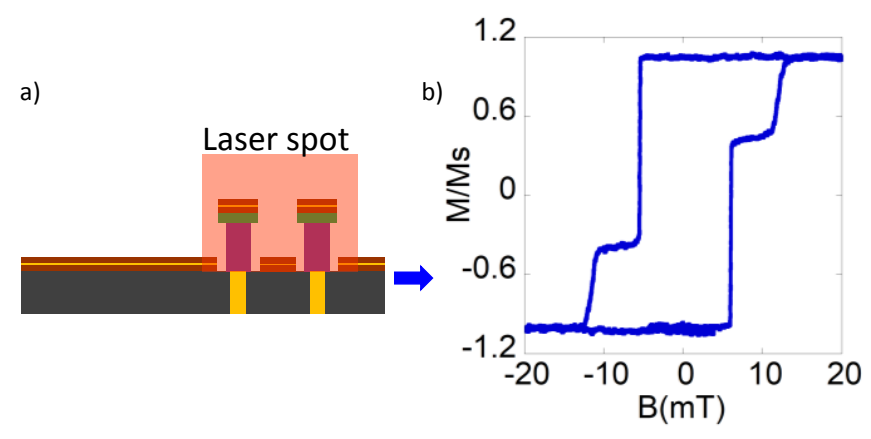

Figure 2: (a) Schematic of the pillars showing the laser spot of the MOKE measurements focused on few pillars. (b) MOKE measurement of the hysteresis loop showing the switching of a few individual pillars. The magnetic field is applied out-of-plane. Both contributions from the free layers of the MTJs on top of the pillars and in the trenches between pillars are visible (reproduced from Ref.8). 


\section{Holography}

The magnetostatic stray field around the pillars, in vacuum, was mapped by off-axis electron holography. The study was first conducted at room temperature, to provide insights on the magnetostatic coupling between neighboring dots as well as between the magnetic deposits on the top of the pillars and in the trenches.

In Figure 3, two magnetic configurations are analyzed. The first after saturating the storage layer in the up direction and the second after saturating the storage layer in the down direction. This is achieved by applying an out-of-plane magnetic field exceeding $1 \mathrm{~T}$ by exciting the objective lens of the microscope along with the specimen tilted by $60^{\circ}$ into the field. Figures $3(\mathrm{a})$ and (b) show the magnetic phase maps. Since the SyAF is a compensated magnetic layer, the observed contrast is mainly due to the stray field from the storage layer. The observation of a similar but inverted contrast of the magnetic phase is consistent with a reversal of the magnetic configuration between the first and second configuration. The phase profiles in Figures 3(c) and (d) reveal an almost constant slope between the pillars, indicating that the magnetic field is relatively homogenous at these locations. Figures 3 (e) and (f) show the field line maps obtained from the cosine of the phase maps with an enhancement of 100 times of the phase. They reveal that the stray field on top of the pillars is vertical in the two configurations, confirming that the magnetization of the storage layer is out-of-plane as expected. The colors allow better visualization of the spatial variation of the magnetic field orientation for the two magnetic configurations. Figures 3(e) and (f) exhibit very similar magnetic flux configurations with opposite orientation, which confirms that we were able to control the reversal of the magnetization of the storage layer inside the microscope. In addition, an antiparallel orientation between the magnetization of the storage layer on top of the pillars and in the trenches is revealed.

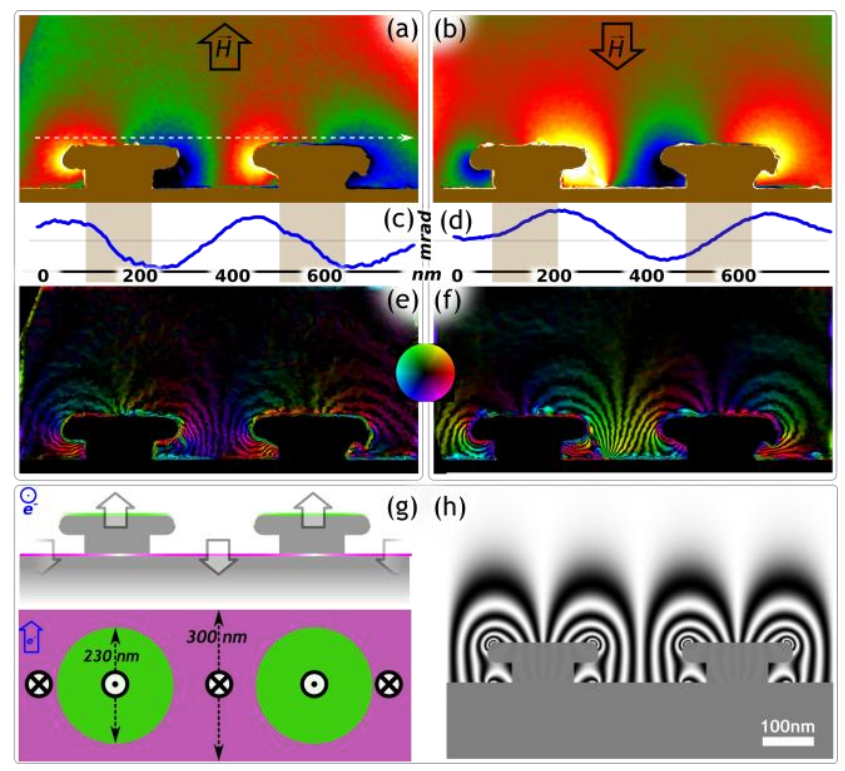

Figure 3: $(a, b)$ Magnetic phase images of the two magnetic configurations (up and down) measured by electron holography. (c,d) Phase profiles obtained $20 \mathrm{~nm}$ above the dots (dashed line in a) for the two configurations. The vertical scale is $\pm 100 \mathrm{mrad}$. (e) and (f) show the colored magnetic induction maps. The vectorial field is encoded in the color wheel where the color depicts the orientation and its saturation the modulus. (g) Schematic of the magnetic configuration used for phase simulation seen from different directions where the direction of electron beam is reported. The top schematic shows the cross-sectional view while the bottom schematic is a top view. (h) The simulated phase shift amplified by a factor of 100 .

Figure 3(h) shows a simulated phase cosine map in presence of such antiparallel configuration as described in Figure 3(g). Figure $3(\mathrm{~h})$ is very similar to the experimentally obtained phase cosine maps (see Figures $3(e, f)$ ). This is true for both directions of the storage layer magnetization. This suggests that in this sample, the deposit in the trenches is sufficiently soft to play the role of a flux absorber thereby reducing the crosstalk between neighboring devices.

The use of the averaging of series of holograms provides a very small phase error of $2.7 \mathrm{mrad}$ which corresponds to an error on the projected magnetic field in the $10^{-11} \mathrm{~T} . \mathrm{m}$ range. This technique is thus expected to be suitable to map the magnetic stray fields of pillars of diameters down to $20 \mathrm{~nm}$ with a good signal-to-noise $(>10)$ even with a magnetic storage layer only $1.4 \mathrm{~nm}$ thick. The magnetic flux at the surface measured is at the centre of each dot. Here we assume an integrated perpendicular flux over its $230 \mathrm{~nm}$ radius. This leads to a magnetic field of $2.5 \mathrm{mT}$ from the $1.4 \mathrm{~nm}$ storage layer. It is worth noticing here that the signal is integrated over the whole electron path along the devices. As the stray field is in the opposite direction above and below each of the dots, this value is underestimated. A previous study on nanowires using cylindrical symmetry assumption and an Abel transformation [14] revealed that the magnetic field measurement was underestimated by approximately $50 \%$ in the nanowire extremity. Applying this to one specific dot leads to a value of the magnetic field at the surface of the dot of $9 \pm 2 \mathrm{mT}$. Regarding the magnetic field in the trench, without such compensation effects in the holography signal, we directly measure a magnetic field of $4.9 \pm 0.5 \mathrm{mT}$. This value is associated to a TEM lamella thickness estimated by scanning electron microscopy observations to be $300 \mathrm{~nm}$. On the contrary to the dot surface, this measurement is less impacted by opposite stray fields at the lamella edge because of the prism geometry which are not measurable due to the lack of cylindrical symmetry.

Further improvements could still be performed to increase the accuracy of the measurements, such as improving the sample preparation to fully remove the protective ink or by depositing a metallic capping over the TEM sample to avoid electrostatic charging effects. Nevertheless despite this charging, the magnetostatic modeling reported in the following section are in agreement with the local experimental observations.

\section{Simulations}

An estimate of the magnitude of the magnetostatic interactions that would exist in MRAM cells of sub- $30 \mathrm{~nm}$ dimensions has been performed. These simulations were 
performed for cell dimensions relevant to STT-MRAM technology. They also provide more quantitative insights on the holographic experimental observations. For this purpose, calculations of the average stray field created by the storage layer of a given cell on the storage layer of a neighboring cell, and on the magnetic deposit at the foot of the pillar were performed. Figure 4(a) shows the results of the simulations that were performed using the Coulomb charged plate method [13]. For these simulations, square pillars with a height of $25 \mathrm{~nm}$ with a $2 \mathrm{~nm}$ thick storage layer having a uniform magnetization of $10^{6} \mathrm{~A} / \mathrm{m}$ were used. This is a good order of magnitude for FeCoB alloys used in MRAM [1]. The dimensions used in the calculations are not exactly the same as the devices observed here, (square versus round shape, FeCoB layer thickness of $2 \mathrm{~nm}$ versus $1.4 \mathrm{~nm}$ ), for calculation purpose, however some semi-quantitative insights can be obtained. Figure 4(b) shows that the vertical average stray field on a square strip of width $\mathrm{F}=100 \mathrm{~nm}$ (approaching half of the pillar diameter of holography experiments) around the foot of the pillar is $5.7 \mathrm{mT}$. The simulations suggest that for pillars with larger widths as in the experiments, the average stray field exerted on the magnetic deposit in the trenches is then larger than the coercive field of the magnetic deposit in the trenches (5.8 $\mathrm{mT}$ shown in Figure 2). This is consistent with the experimental observation that the magnetization in the trenches switches along the stray field originating from the storage layer located on top of the devices, and therefore is aligned antiparallel to it (Figure $3(e, f)$ ).

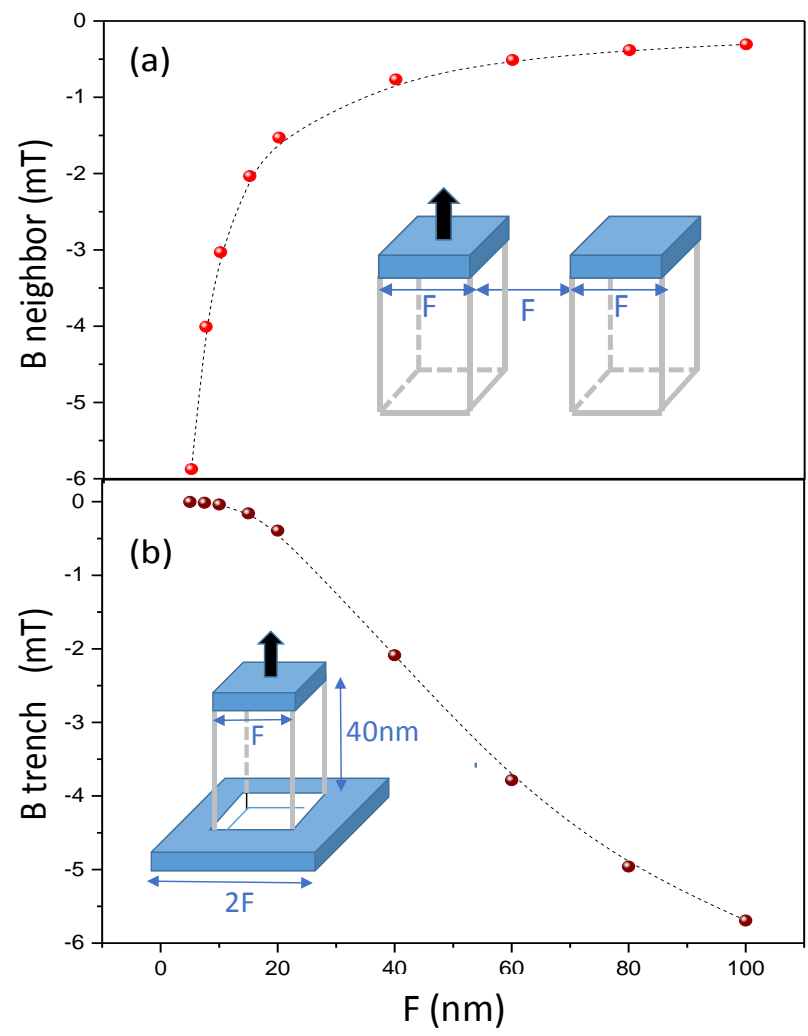

Figure 4: Calculated average stray field from a $2 \mathrm{~nm}$ thick storage layer located on the top of a squared pillar of width F, as indicated in the insets. (a) Stray field exerted on the storage layer of a neighboring cell located on top of a neighboring pillar. The dots are assumed to have an edge to edge spacing of $F$, i.e. the pitch $p=2 F$. The average is calculated on the whole volume of the neighboring storage layer. (b) Stray field exerted on the magnetic deposit at the foot of the pillar. The pillar height is $40 \mathrm{~nm}$. The average is calculated on a square strip of width $F / 2$ surrounding the foot of the pillar.

For cell sizes of $40 \mathrm{~nm}$ and below which are relevant to today's microelectronic technologies, the stray field created by the storage layer of a given cell on the neighboring cell remains of the order of a few mT. This is weak compared to the coercivity of the storage layer material used in state of the art STTMRAM cells which is larger than $200 \mathrm{mT}[1,14]$. This means that the magnetostatic interactions between neighboring cells should not have a too significant impact on the performance of the MRAM array.

\section{Holography combined with in-situ heating}

In-situ annealing holography measurements were performed on the same sample to study the evolution of the magnetic configuration at temperatures up to $325^{\circ} \mathrm{C}$, with $25^{\circ} \mathrm{C}$ increment steps. Figure 5 shows the evolution of the magnetic stray field lines as a function of temperature. The perpendicularly magnetized $1.4 \mathrm{~nm}$ thick storage layer on top of the pillars is found to be stable up to $100^{\circ} \mathrm{C}$, showing outof-plane magnetization. Above this temperature, the magnetization starts to undergo a reorientation from out-ofplane to in-plane. In Figure 5(e), an in-plane configuration is seen at $150^{\circ} \mathrm{C}$ as the field lines connect directly the two pillars. Next from $175^{\circ} \mathrm{C}$ to $275^{\circ} \mathrm{C}$, the magnetic field lines become less dense. This is particularly visible at the bottom of the pillars in Figures $5(f-j)$. It reflects the fact that the magnetization of the storage layer gradually decreases with temperature.

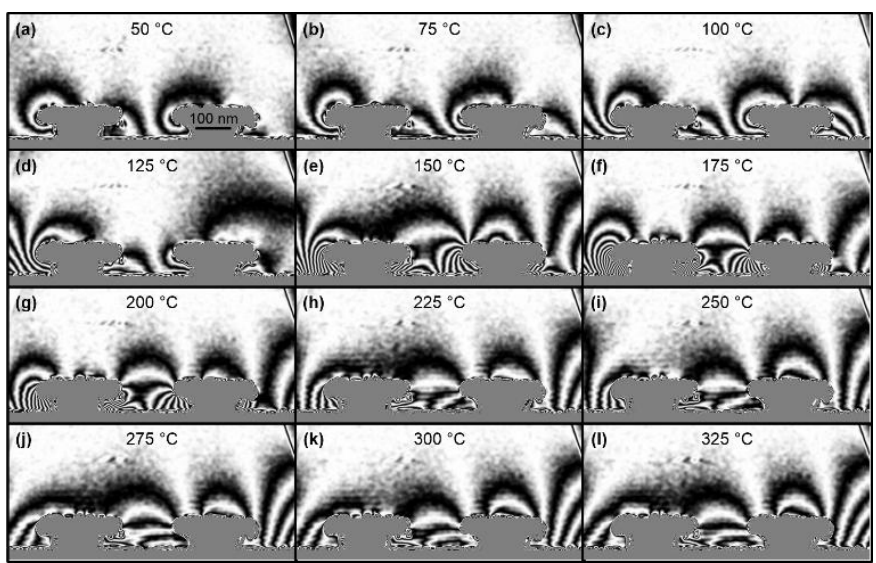

Figure 5: Temperature in-situ holography maps of the stray magnetic field lines in the neighborhood of the pillars, from (a) $50{ }^{\circ} \mathrm{C}$ to (I) $325^{\circ} \mathrm{C}$ with $25^{\circ} \mathrm{C}$ increment steps. Phase information is displayed as the phase cosine amplified by a factor 50 .

Further insight on the micromagnetic configuration of the storage layers can be obtained by modeling the stray field around the MRAM cells. As an example, Figures 6(a) and (b) 
show the experimental results and modeling of the stray field around the dots observed at $150{ }^{\circ} \mathrm{C}$ respectively. Figure 6 (c) shows a schematic used in the simulations which assumes that the left device has still out-of-plane magnetization whereas the right device is in-plane magnetized in a so-called C-state configuration. The experimentally observed stray-field is relatively well reproduced by the simulations. The slight differences between the experimental and simulations that remain may arise from a complex micromagnetic configuration of the magnetic deposit in the trenches.
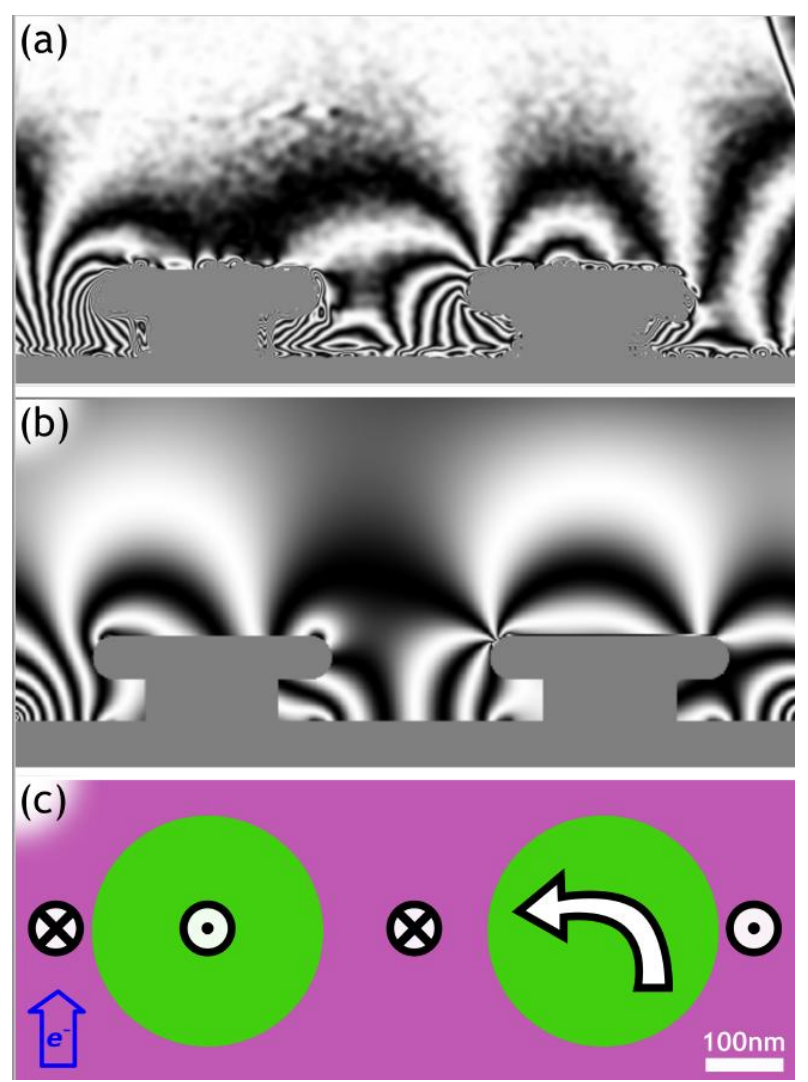

Figure 6: Comparison of the stray fields measured at $150^{\circ} \mathrm{C}$ (a) with the model (b) obtained from the assumed magnetic distribution in the pillar and the trench displayed from top view in (c). The C-state in the right-pillar was modeled using a second-order decrease (from left to right) of the in-plane magnetization. Same material and thickness parameters as in Figure 3 were used as well as a factor of 50 for cosine of phase.

\section{Conclusions}

We have shown that electron holography is a very powerful tool to investigate the micromagnetic behavior of MRAM cells. Estimates show that good signal to noise ratio could still be obtained for cells of diameter of the order of $20 \mathrm{~nm}$. The technique has been applied here to investigate the micromagnetic properties of arrays of MRAM cells prepared by depositing the MTJ material on pre-patterned metallic pillars with undercuts. An antiparallel alignment between the magnetization of the storage layer on top of the dot and around the foot of the pillar was observed indicating flux closure between these two parts of the magnetic deposit. This is an interesting feature since it implies reduced crosstalk between neighboring dots as the magnetic deposit in the trenches plays the role of a magnetic flux absorber (magnetic shield). Our study has shown that insights on the micromagnetic configuration of the storage layer in individual memory cell can be obtained by modelling the stray field surrounding the cell.

We remind that this study was conducted on samples purposely designed so that the storage layer thickness has a relatively weak out-of-plane anisotropy, and correlatively low coercivity. In conventional MTJ for STT-MRAM cells, the storage layer out-of-plane anisotropy would be significantly larger resulting in higher coercivity and an anisotropy reorientation taking place at much higher temperature.

\section{Acknowledgements}

This work was partly funded by the ERC advanced grant MAGICAL $n^{\circ} 669204$, the ERC Starting grant Holoview $N^{\circ} 306535$ and the Carnot project MAGICMAPS. The TEM experiments were performed on the nano-characterization platform at Minatec, Grenoble. The sample were fabricated in the Plateforme Technology Amont (PTA) in Grenoble, from which support from the Renatech network is acknowledged.

\section{Notes and references}

1 D. Apalkov, B. Dieny and J. M. Slaughter, Proceedings of the IEEE, 2016, 104, 1796.

2 L. Thomas, G. Jan, S. Serrano-Guisan, H. Liu, J. Zhu, Y.J. Lee, S. Le, J. Iwata-Harms, R.Y. Tong, S. Patel, V. Sundar, D. Shen, Y. Yang, R. He, J. Haq, Z. Teng, V.Lam, P.Liu, Y.J. Wang, T. Zhong, $\mathrm{H}$. Fukuzawa, and P. Wang, Proceedings of the 2018 IEDM conference, 2018, 27.3.

3 S.-W. Chung, T. Kishi, J.W. Park, M. Yoshikawa, K. S. Park, T. Nagase, K. Sunouchi, H. Kanaya, G.C. Kim, K. Noma, M. S. Lee, A. Yamamoto, K. M. Rho, K. Tsuchida, S. J. Chung, J. Y. Yi, H. S. Kim, Y.S. Chun, H. Oyamatsu and S. J. Hong, Proceedings of the 2016 IEDM conference, 2016, 27.1.

4 N. Perrissin, S. Lequeux, N. Strelkov, A. Chavent, L. Vila, L. D. Buda-Prejbeanu, S. Auffret, R. C. Sousa, I. L. Prejbeanu and B. Dieny, Nanoscale, 2018, 10, 12187.

5 K. Watanabe, B. Jinnai, S. Fukami, H. Sato \& H. Ohno, Nature Communications, 2018, 9, 663.

6 Y. Kim, S. C. Oh, W. C. Lim, J. H. Kim, W. J. Kim, J. H. Jeong, H. J. Shin, K. W. Kim, K. S. Kim, J. H. Park, S. H. Park, H. Kwon, K.H. Ah, J. E. Lee, S. O. Park, S. Choi, H. K. Kang, C. Chung, 2011 Symposium on VLSI Technology Digest of Technical Papers, 2011, 11B-1.

7 M. Gaidis, "Magnetic back-end technology" in "Introduction to Magnetic Random Access Memories", IEEE Press Wiley, 2017, ISBN 111900974X, 9781119009740.

8 V.D. Nguyen, P. Sabon, J. Chatterjee, L. Tille, P.V. Coelho, S. Auffret, R. Sousa, L. Prejbeanu, E. Gautier, L. Vila, and B. Dieny, Proceedings of the 2017 IEDM conference, 2017, 38.5.

9 A.Tonomura, T. Matsuda, J. Endo, T. Arii, and K. Mihama, Phys. Rev. Lett. , 1980, 45, 1430.

10 V. Boureau, R. McLeod, B. Mayall and D. Cooper, Ultramicroscopy, 2018, 193, 52-63.

11 R.E. Dunin-Borkowski, T. Kasama, A. Wei, S.L. Tripp, M.J. Hÿtch, E. Snoeck, R.J. Harrison and A. Putnis, Microscopy Research and Technique, 2004, 64, 390-402.

12 C Phatak, L.de Knoop, F. Houdellier, C. Gatel, M. Hÿtch and A. Masseboeuf, Ultramicroscopy, 2016, 164, 24-30. 
13 Magnetism Fundamentals, edited by E. du Tremolet de Lacheisserie, D.Gignoux and M.Schlenker, Springer, 2003.

14 J.J. Kan, C. Park, C. Ching, J. Ahn, L. Xue, R. Wang, A. Kontos, S. Liang, M. Bangar, H.Chen, S.Hassan, S. Kim, M. Pakala, and S. H. Kang, Proceedings of the 2016 IEDM conference, 2016, 27.4. 